Running head: ATTENTION AFFECTS MOOD

\title{
Changes in the breadth of visual-spatial attention affect subsequent mood
}

Asma Hanif \& Mark J. Fenske

University of Guelph

Keywords: Attentional scope, Mood; Global/local; Visual attention; Cognition-emotion interactions

Words in abstract: 136

Words in main text body: 3915

Number of references: 60

\section{Corresponding Author:}

Mark J. Fenske, PhD

Department of Psychology

University of Guelph

Guelph, Ontario, Canada N1G 2W1

Phone: 519-824-4120 Ext. 56411

Email: mfenske@uoguelph.ca

\section{Acknowledgement:}

This work was supported by the Natural Science and Engineering Research Council of Canada, the Canada Foundation for Innovation, and the Ontario Ministry of Research and Innovation. 


\begin{abstract}
Changes in mood can influence the allocation of attention. Positive affect, for example, is often associated with a broadening of attention, whereas negative affect is often associated with a narrowing of attention. Here we examine whether the link between mood and visuospatial attention also works in the opposite direction. Can changes in the breadth of attention affect subsequent mood? We assessed mood both before and after a global/local visual-perception task that required participants to adopt a relatively broad, neutral, or narrow attentional focus. We found that the Broad-focus task resulted in mood ratings that were higher in both affective valence and arousal than those following the Narrow-focus task and Neutral-focus task. These results build on prior findings to show that changes in the focus of attention can have affective consequences that include altered valence and arousal.
\end{abstract}




\section{Changes in the breadth of visual-spatial attention affect subsequent mood}

Changes in affective states have been shown to influence several aspects of cognitive processing. An increase in positive affect, for example, can broaden the scope of attention resulting in increased sensitivity to a wider array of information from the environment (e.g., Clore, Schwarz, \& Conway, 1994; Fenske \& Eastwood, 2003; Fredrickson, 1998; Rowe et al., 2007), especially for self-related information (Grol, Koster, Bruyneel \& Raedt, 2014). Such results reflect a general class of findings — one that has become a major focus of research within experimental psychology and neuroscience - regarding the impact of emotion on attention and other aspects of cognitive control (e.g., for reviews see Yiend, 2009; Pessoa, 2009). But research over the past two decades have made it clear that emotion-attention effects can also be observed in the opposite direction, with variations in attention determining subsequent emotional response (e.g., Raymond et al., 2003; Srinivasan \& Hanif, 2010 - for review see Fenske $\&$ Raymond, 2006). The majority of research into the affective consequences of visual attention has focused on stimulus-specific emotional responses. Here we extend this approach to the more general domain of mood. Given that mood can affect the breadth of attention, can changes in the breadth of attention also affect subsequent mood?

Support for our hypothesis that mood can be influenced by changes in the breadth of attention can be found in studies of associative thinking. As mood becomes more positive it also leads individuals to describe autobiographical events in ways that reflect more abstract and global representations (Beukeboom \& Semin, 2005) and increases the processing of relations among remotely-associated stimuli (Isen \& Daubman, 1984).

Thus, the relation between mood and breadth of processing is not limited to the allocation 
of visual-spatial attention but also modulates the level of associative processing in higherlevel cognition. Based on the observation that the neural regions supporting these types of effects are massively and reciprocally interconnected, Bar (2009) proposed that not only should positive mood promote associative thinking, but that associative thinking should also promote positive mood. As found in subsequent research, participants' mood is indeed enhanced after word-based tasks that increase the breadth and level of associative thought (Mason \& Bar, 2012; Brunyé, Gagnon, Paczynski, Shenhav, Mahoney, \& Taylor, 2013). If the mechanisms that govern the breadth of internal cognitive-associative processing are similar to those that govern the breadth of external visual-spatial processing, then similar mood effects might be found when breadth of processing is manipulated through changes in visual-spatial attention.

There is growing evidence that the mechanisms that govern the selection of internal mental representations for further processing (i.e., information represented in the absence of external sensory stimulation) are indeed similar to those that govern the selection of external sensory information (for review see Chun, Golomb, \& TurkBrowne, 2011). The breadth of associative thought, for example, is thought to be mediated by the extent to which inhibitory signals originating in prefrontal cortex suppress the activation of other cortical regions that represent concepts and their associations (e.g., Bar, 2009). A narrowed focus is therefore achieved by inhibiting everything except the information that is most directly related to current goals, whereas a broader focus is achieved by relaxing the reins of cognitive control and allowing the activation of more distantly-related representations. Accordingly, changes in affect may modulate the strength and focus of these inhibitory signals and thereby account for 
findings such as positive mood leading to increased memory for irrelevant information (Biss \& Hasher, 2011) and the generation of increasingly unusual word associations (e.g., Isen et al., 1985). Importantly, behavioural and electrophysiological evidence that mood affects the breadth of visual-spatial processing - even in early visual areas - has been interpreted as resulting from the affective modulation of prefrontal inhibitory signals (Vanlessen, Rossi, De Raedt \& Pourtois, 2013). Thus, the way in which mood affects the breadth of visual-spatial attention — through changes in inhibitory signals - may be similar to the way in which it affects the breadth of cognitive-associative processing.

To date, there is only limited evidence that mood can, in a reciprocal fashion, be affected by changes in the breadth of visual-spatial attention, just as it can be by changes in cognitive-associative processing. Both Gu et al. (2017) and Ji et al. (2019), for example, found that tasks that encourage broadened perceptual processing (e.g., viewing movie clips that zoomed out to reveal a broader aspect of each scene) - but not those that encourage narrowed perceptual processing (e.g., viewing movie clips that zoomed in on a subset of each scene) — can be effective in alleviating negative moods that were initially induced in participants by having them watch a sad movie clip. However, while these results may be due to a direct increase in positive affect due to broadened attention, it is also possible that broadening attention alleviated negative mood in these studies by increasing the breadth of thought and thereby reducing rumination on the aspects of the sad movie that was causing the negative mood in the first place. Thus, our investigation examined the effect on mood of changes in the breadth of attention without an initial mood-induction procedure. 


\section{Present study}

We used a global/local visual perception task to manipulate the breadth of visualspatial attention and measured self-reported mood before and after each attentional manipulation. Our choice of attention task was based on the fact that much of the work examining the broadening and narrowing of the focus of attention has utilized hierarchical stimuli within tasks that vary global versus local visual processing (e.g., Srinivasan \& Hanif, 2010; Hanif et al., 2012). A typical hierarchical stimulus consists of a large form (the global dimension) composed of many smaller forms (the local dimension). Participants in our experiment were therefore instructed to identify either the global form or local detail of hierarchical stimuli to induce a broad or narrow focus of attention, respectively (see Navon, 1977). Mood was assessed using an affect grid (Russell, Weis \& Mendelsohn, 1989), which allowed participants to quickly indicate their present affective state in terms of both valence (from negative to positive) and arousal (from low to high). We predicted that the mood of participants would be better (higher valence and arousal) after maintaining a broad focus of attention than after maintaining a narrow focus of attention. To examine whether the 'broadening' or 'narrowing' of attention is more critical for determining subsequent mood, participants also completed a separate neutral-focus attention condition, which involved identifying the perceptual forms of individual non-hierarchical stimuli.

\section{Methods}

\section{Participants}

Forty-three (26 women; mean age: 20.1 years) University of Guelph introductory psychology students took part in the experiment in exchange for course credit. The 
appropriateness of our sample size was determined using $\mathrm{G}^{*}$ Power (Faul, Erdfelder, Lang, \& Buchner, 2007), which indicated that 33 participants would be required to detect the same-sized effect of attentional-breadth manipulation as obtained in Hanif et al.'s (2012) Experiment $2(d=.68)$ with a power of 0.95 at an alpha of .05 . All participants had normal or corrected to normal vision. Informed consent was obtained in compliance with procedures set by the University of Guelph Research Ethics Board.

\section{Stimuli}

For the global precedence task, which served as a measure of individual differences in attentional focus or dispositional biases to attend to global or local information, hierarchical Navon-type letter stimuli were used. The global and local levels of these stimuli were either congruent (e.g., a big 'H' composed of small 'H's) or incongruent (e.g., big 'H' made of small 'S's). A different set of hierarchical stimuli was used to induce a broad or narrow focus of attention in the attentional manipulation task. These stimuli were shapes (triangle, square, circle, diamond) instead of letters, and they were always incongruent (e.g., a large triangle composed of small squares). For all hierarchical stimuli, the global level of the stimuli measured approximately $7.2 \mathrm{~cm} \mathrm{X}$ $7.2 \mathrm{~cm}$ and the local level approximately $0.9 \mathrm{~cm} \times 0.9 \mathrm{~cm}$. Two separate pilot studies were conducted to ensure that these stimuli lacked global or local precedence and were equivalent in terms of task difficulty. For a neutral-focus control condition, a set of nonhierarchical shapes measuring approximately $2.94 \mathrm{~cm} \mathrm{X} 2.94 \mathrm{~cm}$ in size were used. Compared to the hierarchical stimuli, these control items were smaller than the global dimension, larger than the local dimension, and lacked any global-local interference 
(Figure 1). All stimuli were black presented on a white background at a viewing distance of $60 \mathrm{~cm}$.

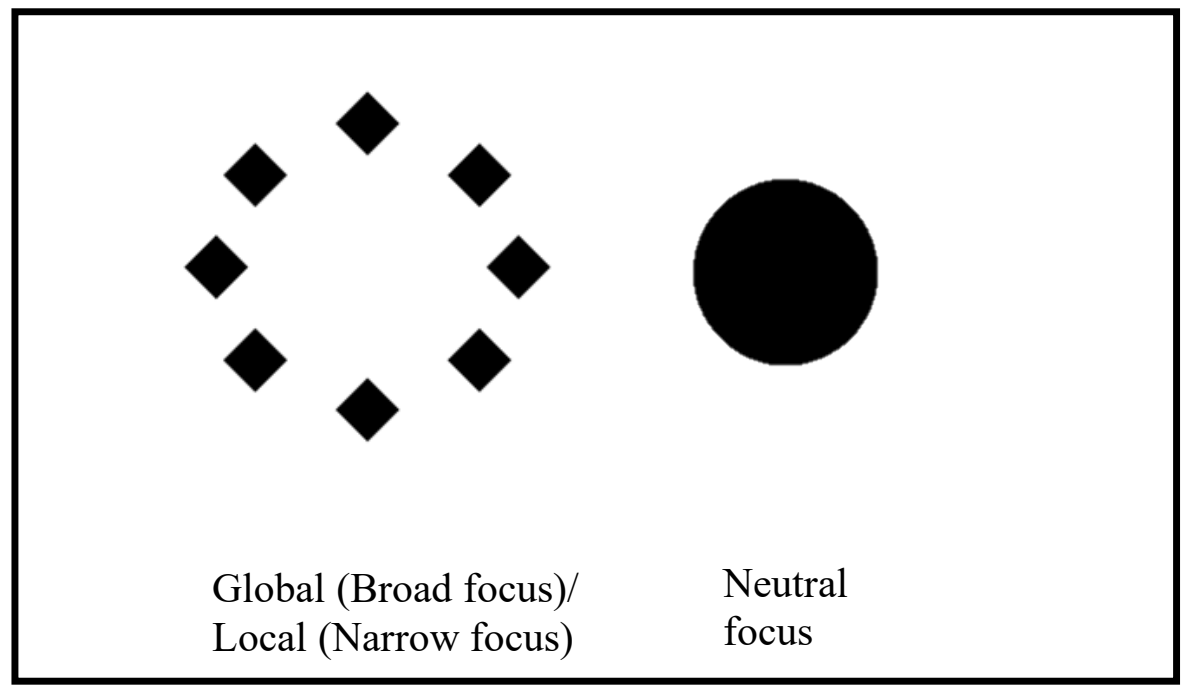

Figure 1. Hierarchical stimuli

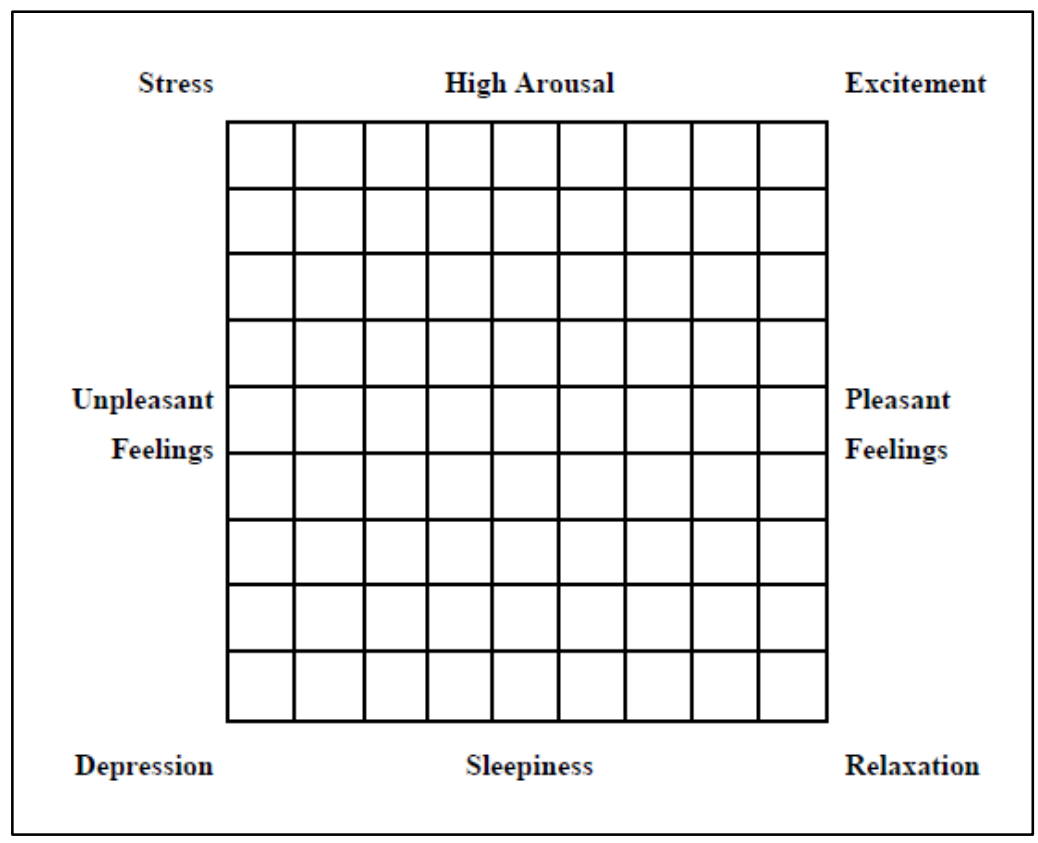

Figure 2. Affect Grid: Horizontal dimension represent valence, vertical dimension represent arousal. 


\section{Design \& Procedure}

Participants first completed the assessment of global precedence. This was comprised of four alternating blocks (24 trials each) in which participants identified either the big letter (requiring global processing), or the small letter (requiring local processing) of the hierarchical stimuli. In each block, congruent and incongruent stimuli were presented with equal probability, for $200 \mathrm{~ms}$ each, followed by a blank screen until response. Participants made speeded key presses (H or S) to identify the target letter. The main part of the experiment followed immediately after the global-precedence assessment.

In the main part of the experiment, participants were asked to provide an initial rating of their current mood (Pre-task: Baseline) and again after each of the three attention-manipulation tasks (Post-task: Broad-focus, Neutral-focus, and Narrow-focus). Mood ratings were made using a 9x9 affect grid (Russell, Weis \& Mendelsohn, 1989), with the x-axis labeled for valence (unpleasant to pleasant) and the y-axis labeled for arousal (low arousal / sleepiness to high arousal). The order of the Broad-focus, Neutralfocus and Narrow-focus attentional manipulation tasks were counterbalanced across participants.

Each trial of the attention-manipulation tasks started with a fixation cross for $500 \mathrm{~ms}$, followed by a hierarchical stimulus for $200 \mathrm{~ms}$, followed by blank screen. Participants were asked to identify either the big shape (Broad-focus task), the small shape (Narrow-focus task), or the only shape (Neutral-focus task). Participants identified the target shapes by pressing keys ' $\mathrm{N}$ ' or ' $\mathrm{M}$ ' (labeled as 'circle' and 'triangle': Broadfocus task), keys ' $V$ ' or 'B' (labeled as 'square' and 'diamond': Narrow-focus task), or 
keys 'N', 'M', 'V' or 'B' (labeled as 'circle', 'triangle', 'square' and 'diamond': Neutralfocus control task). In the neutral-focus task, each participant identified either circles and triangles or squares and diamonds.

\section{Results}

Our main measures of interest were the mood-related valence and arousal scores obtained after the attentional-manipulation tasks (Post-task: Broad-focus, Neutral-focus, and Narrow-focus). A 2 (Mood-measure: Valence, Arousal) x 3 (Task: Broad-focus, Neutral-focus, Narrow-focus) repeated-measures ANOVA revealed that our manipulation of attentional breadth had a significant effect on subsequent mood [Task main effect: $\left.F(2,84)=8.06, p=0.0006, \eta^{2}=.161\right]$, and that this effect was similar for ratings of valence and arousal (Task x Mood-measure interaction: $F(2,84)=0.12, p=0.89, \eta^{2}=$ .003]. Separate 2 x 2 repeated-measures ANOVAs with Task and Mood-measure as factors revealed that this effect of attentional breadth was characterized by higher mood ratings following the Broad-focus task than the Neutral-focus task, $F(1,42)=9.03, p=$ $0.004, \eta^{2}=.177$, and that this effect was similar for ratings of valence and arousal (Task x Mood-measure interaction: $\left.F(1,42)=0.23, p=0.63, \eta^{2}=.006\right)$. Mood ratings were also higher following the Broad-focus task than the Narrow-focus task, $F(1,42)=15.26, p=$ $0.0003, \eta^{2}=.267$, and that this effect was similar for ratings of valence and arousal (Task x Mood-measure interaction: $\left.F(1,42)=0.32, p=0.86, \eta^{2}=.001\right)$. In contrast, there was no difference in mood ratings following the Neutral-focus and Narrow-focus tasks, $F(1,42)=0.16, p=0.69, \eta^{2}=.004$. In sum, maintaining a broad focus of attention resulted in mood states that were more positive in valence and higher in arousal than those elicited by maintaining a neutral or narrow focus of attention. 
While a broadened focus of attention led to enhanced mood relative to that following a neutral or narrow focus of attention, it is important to note that it did not enhance mood relative to the pre-task baseline. Indeed, inspection of Figure 3 shows that the attentional-manipulation tasks generally produced a decrease in mood ratings, with significant mood decrements produced by the Neutral-focus task, $F(1,42)=7.30, p=$ $0.01, \eta^{2}=.148$, and the Narrow-focus task, $F(1,42)=11.04, p=0.002, \eta^{2}=.208$. In contrast, this general task-induced decrease in mood did not occur for the Broad-focus task, $F(1,42)=0.01, p=0.94, \eta^{2}=0.0001$

The preceding analyses make clear that our manipulation of attentional breadth led to significant changes in subsequent mood. However, it is important to consider whether such changes are due to direct effects of broadening or narrowing attention on mood, or may be due instead to differences in the difficulty of the different tasks or to a potential mismatch between task demands and individual differences in ability to perform global or local perceptual tasks.

To assess these possibilities, we quantified each participant's tendency to adopt a broader or narrower focus of attention by examining their performance on the globalprecedence task. Global precedence scores were computed by subtracting local interference scores (i.e., global-congruent RT minus global-incongruent RT) from global interference scores (i.e., local-congruent RT minus local-incongruent RT). To rule out the possibility that any differences in mood across conditions were due to relative ease of processing associated with individual differences in global/local processing, we examined correlations between global-precedence scores and both pre-task (baseline) valence and arousal: neither was significant $\left(r_{\text {valence }}=-.08, \mathrm{p}=.60 ; r_{\text {arousal }}=.04, \mathrm{p}=.79\right)$. To further 
rule out the possibility that any differences in mood across attention conditions were due differences in task difficulty, we compared participants' mean reaction times and accuracy scores using separate repeated-measures ANOVAs with Task (Broad-focus, Neutral-focus, and Narrow-focus) as a one-way factor (see Table 1 for means). This revealed that the difficulty of the tasks were comparable to the extent that manipulating the breadth of attention did not significantly affect $\mathrm{RTs}\left(F(2,84)=1.49, p=0.23, \eta^{2}=\right.$ .03 ) or accuracy $\left(F(2,84)=1.69 ; p=0.19, \eta^{2}=.04\right)$.

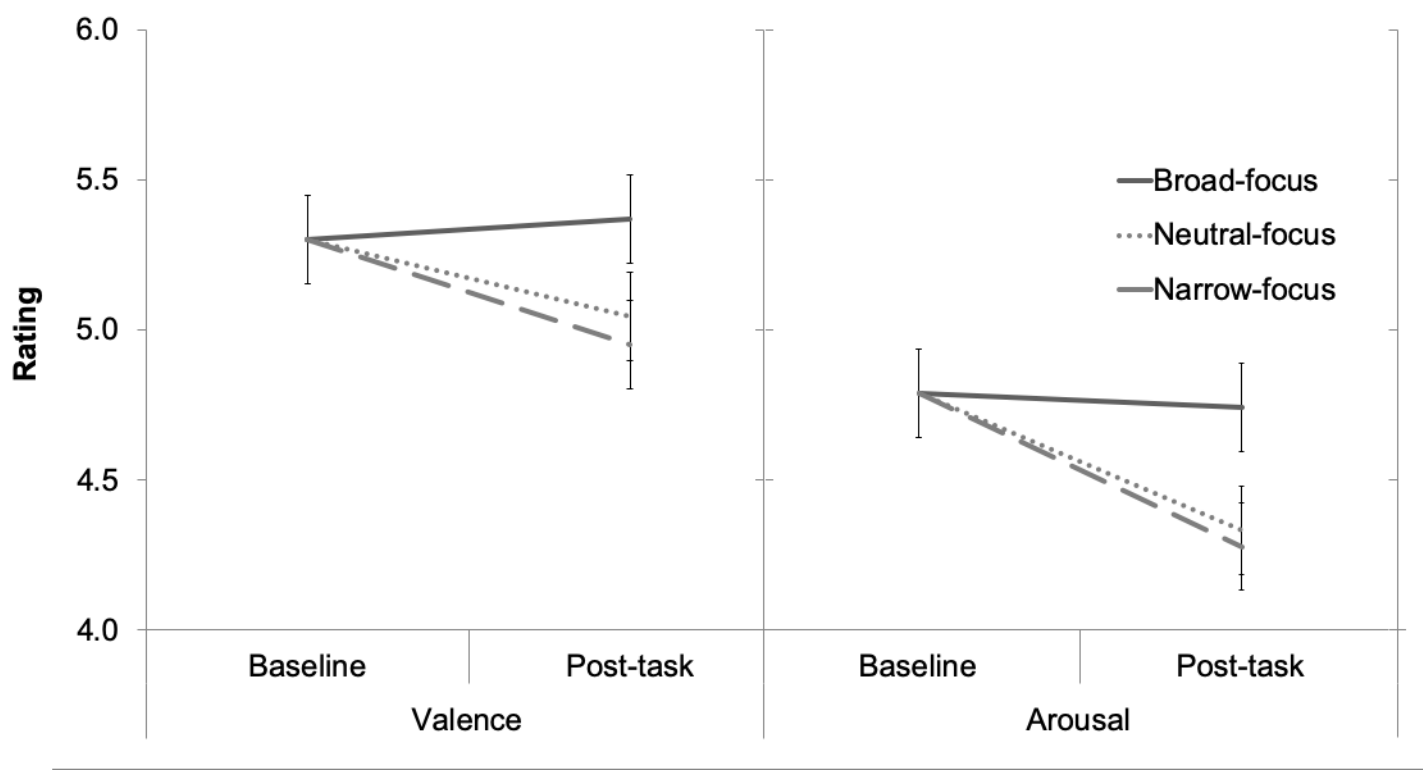

Figure 3: Mean valence and arousal ratings pre- and post- attentional manipulation tasks

\begin{tabular}{|c|c|c|c|c|c|c|c|}
\hline Mood task & Baseline & \multicolumn{2}{|c|}{ Broad-focus } & \multicolumn{2}{c|}{ Neutral-focus } & \multicolumn{2}{c|}{ Narrow-focus } \\
\hline Valence & $5.3(1.83)$ & \multicolumn{2}{|c|}{$5.37(1.36)$} & \multicolumn{2}{|c|}{$5.05(1.66)$} & \multicolumn{2}{|c|}{$4.95(1.52)$} \\
\hline Arousal & $4.79(1.71)$ & \multicolumn{2}{|c|}{$4.74(1.74)$} & \multicolumn{2}{|c|}{$4.28(1.62)$} & \multicolumn{2}{|c|}{$4.28(1.66)$} \\
\hline Attention task & & RT & Accuracy & RT & Accuracy & RT & Accuracy \\
\hline & & $690.19(68.38)$ & $96.23(2.77)$ & $674.97(93.75)$ & $95.28(4.17)$ & $667.43(84.55)$ & $95.72(2.97)$ \\
\hline
\end{tabular}

Table 1. Means and standard deviations (in parentheses) for the mood ratings and attention-task performance for each experimental condition. 


\section{Discussion}

This experiment was conducted to investigate the influence of attentional manipulations on mood. Consistent with our predictions, Broad-focus induced by global processing resulted in better mood as compared to Narrow- and Neutral-focus. Although there was no significant enhancement of valence and arousal by the Broad-focus task as compared to the baseline mood, Broad-focus did result in mood enhancement when compared with Narrow-focus and Neutral-focus task. On the other hand, Narrow-focus of attention seemed to impair mood when compared to the baseline mood. It is important to note that cognitive tasks that require self-sustained attention commonly result in negative mood states, such as boredom (e.g., Danckert \& Merrified, 2018; Hunter \& Eastwood, 2018). This is consistent with what we observed in the Neutral- and Narrow-focus tasks. Interestingly, Broad-focus task appeared to counter this effect to result in a relatively better mood.

Taken together, these results are consistent with earlier findings linking positive mood and a broad focus of attention, as well as negative mood and a narrow focus of attention (Frederickson, 2001; Frederickson \& Branigan, 2005; Gasper \& Clore, 2002). Moreover, our findings provide evidence of enhancement of mood with changes in focus of attention. Most studies on mood and attention have shown that mood states influence the focus of attention, but to the best of our knowledge this is amongst the first studies that has shown such a reciprocal effect. Indeed, our results converge with those recently reported by Ji et al. (2019), who also found that global processing resulted in happier mood as compared to local processing, to support the view that inducing a broad or narrow focus of attention directly impacts mood state. 
Given the possibility that inducing a broadened attentional state can enhance mood, it is not surprising that there has also been interest in establishing the capacity of cognitive tasks requiring broadened attention to elevate depressed mood. To that end, Gu et al. (2017) induced sad/depressed mood via a movie-viewing manipulation before having participants complete a scene-viewing task that 'zoomed-out' to promoted broad attention or 'zoomin' to promote narrow attention. They found that broadening attentional scope in this way alleviated the negative mood induced by the movie, whereas narrowing attentional scope led to persisting negative mood. In a second study, $\mathrm{Gu}$ et al. could relieve even mildly depressed mood. It was found that individuals who were induced to feel sad, could return to the baseline mood after having the broadened attention task but not after having the narrowed attention task. Additionally, depressed individuals demonstrated increased positive affect, and decreased negative affect after receiving attention broadening training compared to those who received attention narrowing training.

Over the last two decades there has been a rapid advancement in neuroimaging techniques that have provided information about the cognitive neuroscience of mood disorders. Studies in patients with mood disorders, such as major depression, have identified structural and functional abnormalities in various brain regions (Sheline, 2003, Drevets, 2003, Holthoff et al., 2004). These regions include the hippocampus, amygdala, anterior cingulate, thalamus, ventromedial- and dorsomedial- prefrontal cortex and subgenual cingulate cortex. Interestingly, some of these areas are part of the default mode network $(\mathrm{DMN})$ - task negative brain areas that are activated at rest and deactivated in the presence of cognitively demanding tasks (Raichle et al., 2001; Greicius, Krasnow, Reiss \& Menon, 2003). The DMN consists of hubs in the ventromedial and dorsomedial 
prefrontal cortex, posterior cingulate cortex, and inferior parietal and lateral temporal regions, including hippocampal formation (Buckner et al., 2008). Note that hyperactivation of these areas have been reported in mood disorders (Sheline, 2003, Drevets, 2003). Conversely, attention-demanding tasks activate a task-positive network that is also found to be active during rest in an anti-correlated manner with the DMN (Fox et al., 2005; Fransson, 2005). During performance of attention-demanding cognitive tasks, two opposite types of responses are commonly observed. A specific set of frontal and parietal cortical areas demonstrate increased activation (Cabeza \& Nyberg, 2000; Corbetta \& Schulman, 2002), whereas activity in DMN decreases (Simpson, Snyder, Gusnard \& Raichle, 2001). As the attentional demands of the task increase, this dichotomy becomes more pronounced; activity in task-positive regions is further increased (Wojciulik \& Kanwisher, 1999), whereas activity in negative regions is further decreased (McKiernan, Kaufman, Kucera-Thompson \& Binder, 2003). Reduction of activity in the DMN during cognitively demanding tasks can be interpreted as reflecting the need to attenuate the brain's self-referential activity as a means of more effectively focusing on the task. Buckner et al. (2008) suggested that these anti-correlated networks compete with one another for control of information processing within the brain. A failure to appropriately engage in attention processing might lead to interference in task performance from internal emotional states, as seen in patients with depression. Additionally, attention-based meditation has been proven to increase left-sided anterior activations associated with increase in positive mood and reduction in anxiety and negative mood state (Davidson et al., 2003). Thus, improving attentional processing might be the key to better mood. 
Successful allocation of attention by global processing yields fluent information processing by reducing interference from local information. As Navon's (1977) classic work demonstrated, people have a default tendency to perceive and rely upon global information rather than local information. Global information tends to be naturally dominant both perceptually as well as conceptually (Fiske \& Taylor, 1991). In impression formation studies, individuals have a predispositional tendency to rely on categorical information as a basis for their judgments (Brewer, 1988; Fiske \& Neuberg, 1990). Such a tendency increases with better mood states and individuals with sad mood rely on more detailed behavioral information (Isbell, 2004). Thus, inducing a broad focus of attention with global processing put individuals in a more naturally dominant or 'fluent' state that might induce ease of processing of incoming information. Furthermore, fluency of information processing has been shown to induce positive mood states. Studies suggest that easier and faster information processing elicits a more positive mood, as indicated by self-report, and by signs of smiling in the zygomatic muscle (Winkielman \& Cacioppo, 2001). Thus, broad focus of attention induced by global processing puts an individual in a fluent state that results in positive mood.

Global processing is accompanied with less inhibition from the non-dominant local dimension. On the other hand, local processing is accompanied with more inhibition exerted by the dominant global dimension. Inhibition is related to negative affect. It is to be noted that impaired inhibition of negative emotional information is an important cognitive component of depression (Goeleven, De Raedt, Baert \& Koster, 2006) and depressed individuals show reduced activations in ACC, the brain area responsible for inhibition (Rogers et al., 2004). Thus, narrow focus of attention leads to worse mood than 
broad focus of attention due to high inhibition induced by local processing. Broad attentional focus also prevents narrow attention or "rumination", a hallmark of mood disorders, by directing attention away from narrow negative thought processing and helps to gain a broader perspective.

The relationship between attention and mood has been implicated in various fields of research, but few studies have investigated the impact of attentional manipulations on mood in the laboratory settings. Studies on clinical population with mood dysfunctions have indicated that negative mood states in such populations are a result of poor attentional processing. Depressed individuals show high self-directed attention and persistent narrow attention on negative thoughts or "rumination" (Gross, 1998). They are also slower to disengage their attention from any stimuli while engaged in a stress task (Ellenbogen et al., 2006). Deficit in inhibition of irrelevant information is considered as a “cognitive vulnerability factor” for causing depression (Joormann, 2007). Mood disorders are accompanied with deficits in the brain regions associated with attentional control and hyperactivations in the brain regions associated with emotion processing. PET and fMRI studies have reported decreased activation of cortical regions responsible for attentional control (dorsolateral prefrontal cortex and anterior cingulate cortex) in depression (Ketter, George, Kimbrell, Benson \& Post, 1996; Drevets et al., 1997). Conversely, increased activation of emotion related limbic regions such as the medial thalamus, pallidostriatum and amygdala (Drevets 2000; Mayberg et al 1999; Sheline et al., 2001; Siegle et al., 2002) have been reported in depression. Depression may be caused by the disruption of the cortical-subcortical pathway resulting in decreased attentional control on the limbic areas (Mayberg et al., 1999). These studies support the idea that successful 
engagement of attention related brain areas might be a key to healthy emotional functioning and positive mood. Wells (1990) developed the attentional training (ATT) program for patients with mood dysfunctions. This task trains individuals to reallocate their attention from emotional to neutral information processing through the use of three auditory tasks. By training selective attention, divided attention, and attention shifting mechanisms, participants with depression and anxiety showed improvements in their mood states (Papageorgiou \& Wells, 2000; Siegle, Ghinassi, \& Thase, 2007). Interestingly, these attentional training tasks are 'cue-specific' i.e. training to draw attention away from negative cues and towards positive cues results in enhancement of positive mood but the results of this experiment show that fundamental and cue-invariant attentional processes may be useful in enhancing mood. Additionally, mindfulness-based cognitive therapy, which combines cognitive therapy with mindfulness techniques, has also been successfully used as an intervention to reduce relapses in recurrent major depression (Segal, Williams \& Teasdale, 2002). Bishop et al. (2004) explained that mindfulness involves components of sustained attention, attentional switching, and cognitive control. Thus, efficient attentional mechanisms can be used as means of improving mood states in clinical population. This study provides empirical support for testing the possibility that simple attention-based interventions that manipulate the focus of attention may be useful for enhancing mood. 


\section{References}

Beukeboom, C.J., \& Semin, G.R. (2005). Mood and representations of behavior: The how and why. Cognition \& Emotion, 19, 1242-1251.

Bishop, S. R., Lau, M., Shapiro, S., Carlson, L., Anderson, N. D., \& Carmody, J. (2004). Mindfulness: A proposed operational definition. Clinical Psychology: Science and Practice, 11, 230-241.

Biss, R. K., \& Hasher, L. (2011). Delighted and distracted: Positive affect increases priming for irrelevant information. Emotion, 11(6), 1474.

Brewer J. A., Worhunsky P. D., Gray J. R., Tang Y. Y., Weber J., \& Kober H. (2011). Meditation experience is associated with default mode network activity and connectivity. Proceedings of National Academy of Science, 108, 2025420259.

Brunyé, T. T., Gagnon, S. A., Paczynski, M., Shenhav, A., Mahoney, C. R., \& Taylor, H. A. (2013). Happiness by association: Breadth of free association influences affective states. Cognition, 127(1), 93-98.

Buckner, R.L., Andrews-Hanna, J.R., \& Schacter, D.L. (2008). The brain's default network: Anatomy, function, and relevance to disease. The Year in Cognitive Neuroscience, eds Kingstone A, Miller MB (Blackwell Publishing, Malden, MA), 1-38.

Cabeza, R., \& Nyberg, L. (2000). Imaging Cognition II: An empirical review of 275 PET and fMRI studies. Journal of Cognitive Neuroscience. 12(1), 1-47.

Chun, M. M., \& Wolfe, J. M. (2001). Visual Attention. In B. Goldstein (Ed.), Blackwell Handbook of Perception. Oxford, UK: Blackwell Publishers Ltd. 
Clore, G. L., Schwarz, N., \& Conway, M. (1994). Affective causes and consequences of social information processing. Handbook of social cognition, 1, 323-417.

Corbetta, M., \& Shulman, G.L. (2002). Control of goal-directed and stimulus-driven attention in the brain. Nature Review Neuroscience, 3, 201-215.

Davidson, R. J., Kabat-Zinn, J., Schumacher, J., Rosenkranz, M., Muller, D., Santorelli, S. F., et al. (2003). Alterations in brain and immune function produced by mindfulness meditation. Psychosomatic Medicine, 65, 564-570.

Drevets, W. (2000). Neuroimaging studies of mood disorders. Biological Psychiatry, 48(8), 813-829.

Drevets, W. C., Price, J. L., Simpson Jr, J. R., Todd, R. D., Reich, T., Vannier, M., \& Raichle, M. E. (1997). Subgenual prefrontal cortex abnormalities in mood disorders. Nature, 386(6627), 824.

Drevets, W. C. (2003). Neuroimaging abnormalities in the amygdala in mood disorders. Annals of the New York Academy of Sciences, 985(1), 420-444.

Eastwood, J.A., Frischen, A., Fenske, M.J., \& Smilek, D. (2012). The Unengaged Mind: Defining Boredom in Terms of Attention. Perspectives on Psychological Science, 7(5), 482-495.

Ellenbogen, M. A., Schwartzman, A. E., Stewart, J., \& Walker, C. D. (2006). Automatic and effortful emotional information processing regulates different aspects of the stress response. Psycho-neuroendocrinology, 31, 373-387

Fenske, M.J., \& Eastwood, J.D. (2003). Modulation of focused attention by faces expressing emotion: Evidence from flanker tasks. Emotion, 3, 327-343. 
Fenske, M. J., Raymond, J. E., Kessler, K., Westoby, N., \& Tipper, S. P. (2005). Attentional inhibition has social-emotional consequences for unfamiliar faces. Psychological Science, 16(10), 753-758.

Fiske, S. T., \& Neuberg, S. L. (1990). A continuum model of impression formation, from category-based to individuating processes: Influence of formation and motivation on attention and interpretation. In M. P. Zanna (Ed.), Advances in experimental social psychology. 23, 1-74. New York: Academic Press.

Fiske, S. T., \& Taylor, S. E. (1991). Social cognition (2nd ed.). Social Cognition: How people understand self and others. New York: McGraw-Hill.

Fox, M.D., Corbetta, M., Snyder, A.Z., Vincent, J.L., \& Raichle, M.E. (2006). Spontaneous neuronal activity distinguishes human dorsal and ventral attention systems. Proceedings of National Academy of Science, 103, 1004610051.

Fransson, P. (2005). Spontaneous low-frequency BOLD signal fluctuations: an fMRI investigation of the resting-state default mode of brain function hypothesis. Human Brain Mapping, 26, 15-29.

Fredrickson, B. L. (2001). The role of positive emotions in positive psychology: The broaden-and-build theory of positive emotions. American Psychologist, 3, $218-226$.

Fredrickson, B. L., \& Branigan, C. (2005). Positive emotions broaden the scope of attention and thought-action repertoires. Cognition and Emotion, 19, 313-332

Friedman, R. S., \& Forster, J. (2001). The effects of promotion and prevention cues on creativity. Journal of Personality and Social Psychology, 81, 1001-1013. 
Gasper, K., \& Clore, G. L. (2002). Attending to the big picture: Mood and global versus local processing of visual information. Psychological Science, 13(1), $34-40$.

Goeleven, E., De Raedt, R., Baert, S., \& Koster, E.H.W. (2006). Deficient inhibition of emotional information in depression. Journal of Affective Disorders. 93 (1), $149-157$.

Greicius, M.D., Krasnow, B., Reiss, A.L., \& Menon, V., 2003. Functional connectivity in the resting brain: a network analysis of the default mode hypothesis. Proceedings of National Academy of Science, 100, 253-258.

Grol, M., Koster, E. H., Bruyneel, L., \& De Raedt, R. (2014). Effects of positive mood on attention broadening for self-related information. Psychological Research, 78(4), 566-573.

Gross, J. J. (1998). The emerging field of emotion regulation: An integrative review. Review of General Psychology, 2, 271-299.

Gu, L., Yang, X., Li, L. M. W., Zhou, X., \& Gao, D. G. (2017). Seeing the big picture: Broadening attention relieves sadness and depressed mood. Scandinavian Journal of Psychology, 58(4), 324-332.

Hanif, A., Ferrey, A.E., Frischen, A., Pozzobon, K., Eastwood, J.D., Smilek, D., \& Fenske, M.J. (2012). Manipulations of attention enhance self-regulation. Acta Psychologica. 139, 104-110. 
Holthoff, V.A., Beuthien-Baumann, B., Zundorf, G., Triemer, A., Ludecke, S., Winiecki, P., Koch, R., Füchtner, F., \& Herholz, K. (2004). Changes in brain metabolism associated with remission in unipolar major depression. Acta Psychiatrica Scandinavica, 110,184-194.

Isbell, L. M. (2004). Not all happy people are lazy or stupid: Evidence of systematic processing in happy moods. Journal of Experimental Social Psychology, 40(3), 341-349.

Isen, A. M., \& Daubman, K. A. (1984). The influence of affect on categorization. Journal of personality and social psychology, 47(6), 1206.

Isen, A. M. (2004). Some perspectives on positive feelings and emotions: Positive affect facilitates thinking and problem solving. In A. S. R. Manstead, N. Frijda, \& A. Fischer (Eds.), Feelings and emotions: The Amsterdam symposium (pp. 263-281). Cambridge, England: Cambridge University Press.

Ji, L. J., Yap, S., Best, M. W., \& McGeorge, K. (2019). Global Processing Makes People Happier Than Local Processing. Frontiers in Psychology, 10, 670.

Joormann, J., Yoon, K. L., \& Zetsche U. (2007). Cognitive inhibition in depression. Applied and Preventive Psychology, 12, 128-139.

Ketter, T.A., George, M.S., Kimbrell, T.A., Benson, B.E. \& Post, R.M. (1996). Functional brain imaging, limbic function, and affective disorders. The Neuroscientist, 1 (2), 55-65.

Mason, M.F. \& Bar, M. (2012). The Effect of Mental Progression on Mood. Journal of Experimental Psychology: General. 141(2), 217-221. 
Mayberg, H.S., Liotti, M., Brannan, S.K., McGinnis, S., Mahurin, R.K., \& Jerabek, P.A. (1999). Reciprocal limbic-cortical function and negative mood: converging PET findings in depression and normal sadness. American Journal of Psychiatry, 156, 675-682.

Mayberg et al., 1999. These studies support the idea that successful engagement of attention related brain areas might be a key to healthy emotional functioning and positive mood.

Wells, A. (1990). Panic disorder in association with relaxation induced anxiety: An attentional training approach to treatment. Behavior Therapy, 21, 273-280.

Papageorgiou, C., \& Wells, A. (2000). Treatment of recurrent major depression with Attention Training. Cognitive and Behavioral Practice. 7( 4), 407-413.

McKiernan K.A., Kaufman J.N., Kucera-Thompson J., \& Binder J.R. (2003). A parametric manipulation of factors affecting task-induced deactivation in functional neuroimaging. Journal of Cognitive Neuroscience. 15, 394-408.

Navon, D. (1977). Forest before trees: The precedence of global features in visual perception. Cognitive Psychology, 9, 353-383.

Pessoa, L. (2009). How do emotion and motivation direct executive control? Trends in Cognitive Sciences, 13(4), 160-166.

Raichle, M.E, MacLeod, A.M., Snyder, A.Z., Powers, W.J., Gusnard, D.A., \& Shulman, G.L. (2001). A default mode of brain function. Proceedings of the National Academy of Sciences, 98(2), 676-682 
Raymond, J. E., Fenske, M. J., \& Tavassoli, N. T. (2003). Selective attention determines emotional responses to novel visual stimuli. Psychological Science, $14,537-542$

Rogers, M. A., Kasai, K., Koji, M., Fukuda, R., Iwanami, A., Nakagome, K., ... \& Kato, N. (2004). Executive and prefrontal dysfunction in unipolar depression: a review of neuropsychological and imaging evidence. Neuroscience research, 50(1), 1-11.

Rowe, G., Hirsh, J.B.,\& Anderson, A.K. (2007). Positive affect increases the breadth of attentional selection. Proceedings of the National Academy of Sciences, 104(1), 383-388.

Russell, J.A., Weiss, A., \& Mendelsohn, G.A. (1989). The affect grid: A single-item scale of pleasure and arousal. Journal of Personality and Social Psychology, $57,493-502$

Sheline, Y., Barch, D.M., Donnelly, J.M., Ollinger, J.M., Snyder, A.Z., Mintun, M.A. (2001). Increased amygdala response to masked emotional faces in depressed subjects resolves with antidepressant treatment: an fMRI study. Biological Psychiatry, 50, 651-658.

Sheline, Y. (2003). Neuroimaging studies of mood disorder effects on the brain. Biological Psychiatry, 54, 338-352.

Segal, Z.V., Williams, J.M.G., \& Teasdale, J.D. (2002). Preventing depression: mindfulness-based cognitive therapy. New York: Guilford . 
Siegle, G. J., Ghinassi, F., \& Thase, M. E. (2007). Neurobehavioral therapies in the 21st century: Summary of an emerging field and an extended example of cognitive control training for depression. Cognitive Therapy and Research, 31, 235-262.

Simpson, J. R., Drevets, W. C., Snyder, A. Z., Gusnard, D. A., \& Raichle, M. E. (2001). Emotion-induced changes in human medial prefrontal cortex: II. During anticipatory anxiety. Proceedings of the National Academy of Sciences, 98(2), 688-693.

Srinivasan, N., \& Hanif, A. (2010). Global-happy and local-sad: Perceptual processing affects emotion identification. Cognition \& Emotion, 24, 10621069.

Winkielman, P., \& Cacioppo, J.T. (2001). Mind at ease puts a smile on the face: psychophysiological evidence that processing facilitation elicits positive affect. Journal of personality and social psychology. 81(6), 989.

Wojciulik, E., \& Kanwisher, N. (1999). The generality of parietal involvement in visual attention. Neuron, 23, 747-764. 